\title{
KLEINIAN GROUPS WITH QUOTIENTS OF FINITE VOLUME
}

\author{
Troels Jorgensen and Albert Marden*
}

\section{Introduction}

Consider a discontinuous group $G$ of orientation preserving isometries of hyperbolic 3 -space $\mathbf{H}$. The quotient $\mathbf{H} / G$ is an orbifold. Let us fix a positive number $K$ and denote by $\mathcal{S}$ the set of such groups $G$ for which the volume of $\mathbf{H} / G$ is at most $K$. If $K$ is large enough (for instance, if $K \geq 10$ ), then $\mathcal{S}$ contains infinitely many non-isomorphic torsion-free groups. This phenomenon does not occur in any dimension other than 3 (see [5]). On the other hand, it follows from Borel [1] that the arithmetic groups in $\mathcal{S}$ represent only finitely many isomorphism classes. Our purpose is to prove the following

Theorem. There exists in $\mathcal{S}$ a finite set of groups $\mathcal{G}_{1}, \ldots, \mathcal{G}_{s}$ which are maximal in the sense that each $G \in \mathcal{S}$ is the homomorphic image of at least one $\mathcal{G}_{i}, i \in\{1, \ldots, s\}$. The kernels of these homomorphisms are, when non-trivial, the normal closures of parabolic elements.

This was proved in the spring of 1977. At that time our interest in this topic was further stimulated through conversations with B. Mazur and W. Thurston.

The proof goes as follows. Given a sequence $\left\{G_{n}\right\}$ of non-isomorphic groups from $\mathcal{S}$, we construct from a subsequence a "limit" $G$ with the properties that

1) $G \in \mathcal{S}$

and, with the possible exceptions of a finite number of indices $n \in \mathbf{N}$,

2) there is a homomorphism of $G$ onto $G_{n}$, and

3 ) the number of cusps in $G$ is strictly larger than the number of cusps in $G_{n}$.

There is an upper bound for the number of cusps that a group $G \in \mathcal{S}$ can have. Thus, the groups in $\mathcal{S}$ with the maximal number of cusps represent only a finite number of isomorphism classes. Similarly, those groups in $\mathcal{S}$ which have exactly $j$ cusps and which are not homomorphic images of groups in $\mathcal{S}$ with strictly more than $j$ cusps must fall into finitely many isomorphism classes! The finite list of groups $\mathcal{G}_{1}, \ldots, \mathcal{G}_{s}$ can therefore be selected from $\mathcal{S}$ by first choosing one group from each isomorphism class occurring among the groups with the maximal number of cusps and then, proceeding by decent on the number of cusps, choosing

\footnotetext{
* Supported in part by the National Science Foundation.
} 
one group from each isomorphism class occurring among the groups which have one less cusp than any of those already chosen and which are not homomorphic images of any of those. A more detailed description of these matters will emerge below.

Let us denote by $\mathcal{O}$ the set of hyperbolic orbifolds arising as quotients $\mathbf{H} / G$ of groups $G \in \mathcal{S}$. Mostow's rigidity theorem [17], as extended to groups with cusps in [15], tells us that there is a one-to-one correspondence between isomorphism classes of groups $G \in \mathcal{S}$ and isometry classes of orbifolds $M \in \mathcal{O}$. The geometrical version of the theorem is that $\mathcal{O}$ contains a finite number of orbifolds $M_{1}, \ldots, M_{s}$ which are maximal in the sense that every $M \in \mathcal{O}$ arises as the result of Dehn surgery about the cusps of at least one the $M_{i}$ 's. In the case of hyperbolic manifolds (torsion free groups), this was elegantly treated in Thurston's lecture notes [19]. Also presented there was Gromov's result that Dehn surgery on manifolds reduces the volume. It follows, as Thurston pointed out, that the number of hyperbolic manifolds with a given volume is finite and that the set of all hyperbolic manifolds of finite volume can be well-ordered essentially by the volume. Another study of the volume of hyperbolic manifolds was done by Neumann and Zagier [18]. More recently an elementary development based on a theorem of Schlälli was discovered by Craig Hodgson [7]. His approach works for all orbifolds. That is, Dehn surgery about the cusps of orbifolds reduces volume and hence, at most a finite number of orbifolds can have the same volume. Thus, the set of all hyperbolic orbifolds of finite volume can be well-ordered by the volume.

In the next section we survey the theory of fundamental polyhedra. It is followed by a brief discussion of cusps. In the final section the theorem is proved with further details available in [12] ([12] focuses on infinite volume groups and convergence of regions of discontinuity).

\section{Polyhedra}

To prove the theorem we will make use of several results from the theory of Kleinian groups. First of all we will be considering fundamental polyhedra usually referred to as Dirichlet regions: Given a point $\sigma \in \mathbf{H}$ and an orientation preserving isometry $g$ not fixing $\sigma$, the set of points in $\mathbf{H}$ closer to $\sigma$ than to $g^{-1}(\sigma)$ is a hyperbolic half space, $E(g)$, bounded by a plane, $I(g)$. If $G$ is a discontinuous group of isometries of $\mathbf{H}$ and $\sigma \in \mathbf{H}$ is chosen such that no element of $G$ other than the identity fixes $\sigma$, then the Dirichlet polyhedron for $G$, based at $\sigma$, is the set $P(G)=\bigcap_{g \in G \backslash\{\text { id }\}} E(g)$. It is a convex, locally finite polyhedron with faces lying on some of the planes $I(g)$. Those $g \in G$ for which $I(g)$ meets the boundary of $P(g)$ are called facet transformations. If $g \in G$ is a facet transformation then so is $g^{-1}$ and $g$ maps $I(g) \cap \overline{P(G)}$ into $I\left(g^{-1}\right) \cap \overline{P(G)}$; here $\overline{P(G)}$ denotes the closure in $\mathbf{H}$. Identifying the equivalent points on the boundary of $P(G)$, one obtains a model of the quotient orbifold $\mathbf{H} / G$. The number of facet transformations in $G$ will be denoted by $f(G)$. It depends on $\sigma$ (which we have suppressed) and may be 
infinite. However, whether it is finite or infinite does not depend on the particular choice of base point. More detailed expositions can be found in Beardon [2], Ford [3], Lehner [14], Maskit [16] or [11].

Each maximal cycle of equivalent edges of $P(G)$ gives rise to a relation among the facet transformations realizing the equivalences. Namely, when applied in succession, their product fixes a line and thus has finite order.

According to Poincaré's theorem, the facet transformations generate $G$ and the edge relations form a basis for the relations among these generators. In particular, if $f(G)$ is finite, then this gives a finite presentation for $G$. Conversely, a polyhedron centered at $\sigma$ with assigned facet transformations which satisfy the appropriate edge relations and have proper behavior at infinity is the Dirichlet polyhedron for the (necessarily discrete) group generated by the facet transformations. (For the precise statement, see Maskit [16].)

A proof of Poincaré's theorem appears in [16]. As a corollary we see that any set of groups $\{G\}$ which admit polyhedra $\{P(G)\}$ with a uniformly bounded number of facet transformations can represent only finitely many different isomorphism classes or, equivalently, their quotient orbifolds must, up to isometry, consist of only finitely many kinds.

Another important result is the following (see Wielenberg [21] for a direct proof for hyperbolic space):

Theorem (Selberg, Garland-Raghunathan). The volume of $\mathbf{H} / G$ is finite if and only if the action of $G$ on the sphere at infinity is nowhere discontinuous ( $G$ is of the first kind) and $P(G)$ is finite ( $G$ is geometrically finite).

Thus, in the case of finite volume $P(G)$ has at most a finite number of noncompact ends. Each of these terminates at a parabolic fixed point, stabilized in $G$ by a parabolic subgroup of rank 2 . The full stabilizer may contain elliptic transformations as well.

The following result was proved in [13] and [21] for the torsion-free case:

Injectivity theorem. There exists a positive constant $c$ such that each hyperbolic 3-orbifold $M=\mathbf{H} / G$ contains an imbedded ball of radius $c$.

An explicit estimate of the universal constant $c$ can be found in Waterman [20]. The injectivity theorem allows us to construct fundamental polyhedra, by suitably choosing the base point $\sigma$, which all contain a certain ball. For these polyhedra there will then be a uniform upper bound to the number of boundary facets within a given distance of the base point.

\section{Cusps}

Parabolic elements in $G$ always give rise to non-compact ends in $M=\mathbf{H} / G$ and thus in the fundamental polyhedron $P(G)$. The geometry of these ends is most easily described by using the upper half space model for $\mathbf{H}$ and taking $\infty$ 
as the fixed point. The parabolic element is then a euclidean translation. If a discontinuous group $G$ contains the translation $z \rightarrow z+1$ then each of its elements maps the horosphere of points above height 1 either onto itself or onto a disjoint horosphere (see Beardon [2] or Maskit [16]). Thus, a neighborhood of $\infty$ looks modulo $G$ as it does modulo the stabilizer of $\infty$ in $G$. If the translation by 1 is the shortest among the translations in this stabilizer, then we associate with the fixed point $\infty$ the horospherical neighborhood consisting of points above height 1. Similarly, we associate cannonical neighborhoods to the other parabolic fixed points. It is then easy to see that the cannonical neighborhoods of different points are disjoint. When $\mathbf{H} / G$ has finite volume and $\infty$ is a parabolic fixed point for $G$, the stabilizer of $\infty$ is a so called Bieberbach group, consisting of euclidean translations and rotations. The subgroup of translations has rank 2 and index at most 6 . If the shortest translation has modulus 1 , then the euclidean area of a fundamental parallelogram for the group of translations is at least $\sqrt{3} / 2$. It follows that the hyperbolic volume of the cannonical neighborhood modulo the full stabilizer is at least $\sqrt{3} / 24$. Thus we have the

Proposition. If $\operatorname{vol}(\mathbf{H} / G) \leq K$, that is, if $G \in \mathcal{S}$, then the number of cusps in $G$ is at most $8 \sqrt{3} K$.

\section{Compactness}

Returning to the set $\mathcal{S}$ of groups $G$ whose quotients have volume at most $K$, let us consider a sequence $\left\{G_{n}\right\}_{n \in \mathbb{N}}$ from $\mathcal{S}$ and construct from a subsequence a "limit" $G \in \mathcal{S}$ as promised in the introduction. Since we are interested in the groups only up to isomorphism, it may be assumed that there exists a point $\sigma \in \mathbf{H}$ such that the Dirichlet polyhedra $P\left(G_{n}\right)$ with centre $\sigma$ all contain the universal ball $B_{c}$ of radius $c$ with centre $\sigma$ (we may replace the original $G_{n}$ 's by conjugates, if necessary).

Let $B_{r}$ denote the ball of radius $r$ with centre $\sigma$. Then, for each fixed $r \in \mathbf{R}_{+}$, there is an upper bound, independent of $n \in \mathbf{N}$, for the number of facets of $P\left(G_{n}\right)$ that meet $B_{r}$. This is so because there is a uniform upper bound for the number of distinct images of $\sigma$ contained in any compact subset of $\mathbf{H}$. Thus, we may from $\left\{G_{n}\right\}_{n \in \mathbb{N}}$ select a subsequence, which after relabeling again is denoted by $\left\{G_{n}\right\}_{n \in \mathbb{N}}$, with the property that the sets $P\left(G_{n}\right) \cap B_{r}$ converge with structure. By convergence with structure we understand that not only do the sets $P\left(G_{n}\right) \cap B_{r}$ converge as sets in $\mathbf{H}$, but also, for each $n \in \mathbf{N}$, the uniformly finitely many associated facet transformations converge. By repeatedly taking subsequences, say as $r$ runs through the natural numbers, we obtain at last a subsequence (the diagonal one, say) whose polyhedra converge with structure in all of $\mathbf{H}$. Therefore, the limiting polyhedron $P$ comes equipped with facet pairing transformations and is thus a fundamental polyhedron for the group $G$ generated by these facet transformations (Poincare's theorem, [16]). In fact, $P$ must be the Dirichlet polyhedron for $G$, that is $P=P(G)$. 
Clearly, the volume of $P$ cannot exceed $K$. This, by the theorem of Selberg and Garland-Raghunathan, implies that the number of facets of $P$ is finite and so, by Poincaré's theorem, $G$ is finitely generated, namely by its finitely many facet transformations. Since these facet transformations of $P(G)$ are the limits of facet transformations of the approximating groups $G_{n}$ (or polyhedra $P\left(G_{n}\right)$ ), the convergence of the "diagonal sequence" $\left\{G_{n}\right\}_{n \in \mathbf{N}}$ to $G$ is not only geometrical (polyhedral with structure) but algebraic as well. It follows from [9] or [10] that the natural map from the facet transformations for $G$ to the facet transformations for $G_{n}$ extends to a homomorphism $\varphi_{n}$ of $G$ onto $G_{n}$ as soon as $n \in \mathbf{N}$ is sufficiently large.

It remains to show that $G$ has strictly more cusps than all but finitely many of its approximants. Since the sequence $\left\{G_{n}\right\}_{n \in N}$ (the original one and hence any of the subsequences) consist of non-isomorphic groups, the numbers $f\left(G_{n}\right)$ (of facet transformations) must, as a consequence of Poincaré's theorem, tend to infinity. Since the number of facets meeting any ball $B_{r}$ is uniformly bounded, these more and more facets must appear farther and farther away from the base point $\sigma$, that is, closer and closer to the sphere at infinity. Hence these more and more facets must accumulate towards a necessarily finite set of points on (the boundary of $P$. The limit points are parabolic fixed points on $G$. We will show that they cannot also be limits of parabolic fixed points on the polyhedra $P_{n}$. They are the "new cusps"!

Let $p_{1}, \ldots, p_{k}$ be the parabolic fixed points on $P$ and $S_{1}, \ldots, S_{k}$ their parabolic stabilizers. These groups, $S_{j}$, are free abelian of rank 2 and among the exceptions to Jorgensen's inequality [9]. For sufficiently large $n \in \mathbf{N}$, their images $\varphi_{n}\left(S_{j}\right)$ must also be abelian exceptions to that inequality. Therefore each $\varphi_{n}\left(S_{j}\right)$ is either

1) cyclic,

2) generated by two commuting elements, one elliptic and the other loxodromic, or

3) free abelian of rank 2 .

In the cases 1) and 2) there is a neighborhood of $p_{j}$ on the sphere at infinity which does not contain any parabolic fixed points from $P_{n}$. This is so because the fundamental polyhedron for $\varphi_{n}\left(S_{j}\right)$ approximates that for $S_{j}$ and meets the sphere at infinity in a connected set (there are "no holes" in which old cusps could disappear). One thus has a new cusp.

In case 3$), p_{j}$ has a neighborhood which contains (for $n$ large enough) exactly one parabolic point from each $P_{n}$, namely the fixed point of $\varphi_{n}\left(S_{j}\right)$, and these points converge to $p_{j}$. This is because the polyhedra for $\varphi_{n}\left(S_{j}\right)$ tend to the polyhedron for $S_{j}$. In particular, a parabolic point $p_{j}$ on $P$ which is the limit of more and more faces on $P_{n}$ (as $n$ increases) must be a new cusp.

To show that $G$ has strictly more cusps than any of its sufficiently close approximants, it is no restriction to assume that the approximants have the same 
number of cusps, say $C$. For each $n \in \mathbf{N}$, choose a parabolic fixed point $p_{1, n}$ on $P_{n}$. A subsequence of $\left\{p_{1, n}\right\}$ must converge to one of the points $p_{1}, \ldots, p_{k}$. Call this point $q_{1}$. Next choose on $P_{n}$ a parabolic point $p_{2, n}$ which is not equivalent to $p_{1, n}$ in $G_{n}$. A subsequence of $\left\{p_{2, n}\right\}$ must converge to one of the points $p_{1}$, $\ldots, p_{k}$. Call this point $q_{2}$. By [10] $q_{1}$ and $q_{2}$ must be inequivalent under $G$. Also, both $q_{1}$ and $q_{2}$ must be old cusps, that is, their stabilizers have parabolic images (Case 3). Continuing in this way, we see that each of the $C$ inequivalent cusps in (each) $P_{n}$ are represented as inequivalent old cusps in $G$ by points $q_{1}$, $q_{2}, \ldots, q_{C}$. Since $G$ also has at least one new cusp, it has more than $C$ cusps.

\section{Kernels}

Recall that the homomorphisms $\varphi_{n}: G \rightarrow G_{n}$ came about as extensions of the natural maps from the facet transformations for $G$ to the facet transformation for the $G_{n}$ 's.

There exists a large ball $B_{r}$ which meets all the facets of $P(G)$. Inside this ball each $P\left(G_{n}\right)$ looks combinatorially like $P(G)$ (and geometrically almost like $P(G))$. Therefore, in $B_{r}$, the edge-cycles are identical. The only differences in the presentation for $G$ and the presentations for the $G_{n}$ 's must therefore arise from edge-cycles closer and closer to the sphere at infinity. This means that the "new" relations in each $G_{n}$, relative to the relations in $G$, stem from the creation of new cusps in $G$. Thus, the kernel of each $\varphi_{n}$ is contained in the normal closure of the new parabolic elements in $G$. The details of this argument are in [12].

\section{References}

[1] Borel, A.: Commensurability classes and volumes of hyperbolic 3-manifolds. - Ann. Scuola Norm. Sup. Pisa Cl. (4) 8, 1981, 1-33.

[2] Beardon, A.F.: The geometry of discrete groups. - Graduate Texts in Mathematics 91. Springer-Verlag, New York-Berlin, 1983.

[3] Ford, L.R.: Automorphic functions, 2nd edition. - Chelsea, New York, 1951.

[4] Gromov, M.: Manifolds of negative curvature. - J. Differential Geom. 13, 1978, 223-330.

[5] Gromov, M.: Hyperbolic manifolds (according to Thurston and Jørgensen). - Bourbaki Seminar Vol. 1979/80, Lecture Notes in Mathematics 842. Springer-Verlag, BerlinNew York, 1981, 40-53.

[6] Gromov, M.: Volume and bounded cohomology. - Inst. Hautes Études Publ. Math. 56, 1982, 213-307.

[7] Hodgson, C.D.: Degeneration and regeneration of geometric structures of three-manifolds. - Thesis, Princeton University, 1986.

[8] Jorgensen, T.: On cyclic groups of Mobius transformations. - Math. Scand. 33, 1973, 250-260.

[9] Jorgensen, T.: On discrete groups of Mobius transformations. - Amer. J. Math. 98, 1976, 739-749.

[10] Jorgensen, T., and P. KLeIn: Algebraic convergence of finitely generated kleinian groups. - Quart. J. Math. Oxford Ser. (2), 33, 1982, 325-332. 
[11] Jorgensen, T., and A. MARDEn: Generic fundamental polyhedra for kleinian groups. - Holomorphic functions and moduli I. (D. Drasin, C.J. Earle, F.W. Gehring, I. Kra, A. Marden, editors), Mathematical Sciences Research Institute Publications 10. Springer-Verlag, Berlin-Heidelberg-New York, 1988.

[12] Jorgensen, T., and A. MARDEN: Algebraic and geometric convergence of kleinian groups. - Mathematical Sciences Research Institute report 10003319-86.

[13] Jorgensen, T., and A. Marden: Some universal properties of kleinian groups. - To appear.

[14] Lehner, J.: Discontinuous groups and automorphic functions. - Math. Surveys Monogr. VIII, Amer. Math. Soc., Providence, R.I., 1964.

[15] Marden, A.: The geometry of finitely generated kleinian groups. - Ann. of Math. 99, $1974,383-402$.

[16] MASKIT, B.: Kleinian groups. - Grundlehren der mathematischen Wissenschaften 287. Springer-Verlag, Berlin-Heidelberg-New York, 1988.

[17] Mostow, G.D.: Quasiconformal mappings in $n$-space and the rigidity of hyperbolic space forms. - Inst. Hautes Études Publ. Math. 34, 1968, 53-104.

[18] NeumanN, W.D., and D. Zagier: Volumes of hyperbolic 3-manifolds. - Topology 24, 1985, 307-332.

[19] Thurston, W.P.: The geometry and topology of 3-manifolds. - Princeton University lecture notes.

[20] Waterman, P.L.: An inscribed ball for kleinian groups. - Bull. London Math. Soc. 16, 1984, 525-530.

[21] Wielenberg, N.J.: Discrete Möbius groups: fundamental polyhedra and convergence. Amer. J. Math. 99, 1977, 861-877.

Columbia University

Department of Mathematics

New York, NY 10027

U.S.A.

Received 26 July 1988
University of Minnesota

School of Mathematics

Minneapolis, MN 55455

U.S.A. 